\title{
Ensino Sem Erro e Aprendizagem de Discriminação
}

\author{
Raquel Maria de Melo ${ }^{1}$ \\ Departamento de Processos Psicológicos Básicos da Universidade de Brasilia, \\ Brasília, Distrito Federal, Brasil \\ João dos Santos Carmo \\ Departamento de Psicologia da Universidade Federal de São Carlos, \\ São Carlos, São Paulo, Brasil \\ Elenice S. Hanna \\ Departamento de Processos Psicológicos Básicos da Universidade de Brasília, \\ Brasília, Distrito Federal, Brasil
}

\begin{abstract}
Resumo
O termo aprendizagem sem erro tem sido utilizado para se referir, de maneira ampla, à programação de contingências de ensino que resultam em desempenhos precisos ou com pouco erro. A ocorrência de erros durante a aprendizagem tem sido considerada uma parte importante e necessária à aprendizagem na cultura e no meio acadêmico. Na Análise do Comportamento os erros e suas implicações para a aprendizagem são investigados em estudos sobre controle de estímulos e os resultados indicam que erros podem interferir na precisão de desempenhos já aprendidos, gerar comportamentos emocionais, esquiva da tarefa e/ou do professor. Neste estudo, são apresentados diferentes procedimentos de modificação gradual de estímulos para ensinar discriminações, que podem gerar aprendizagem sem erro, e os fatores que afetam a eficácia dessas contingências de ensino. Esses procedimentos necessitam ser mais amplamente divulgados, por serem contribuições importantes da Análise do Comportamento para contextos aplicados, especialmente para o desenvolvimento de tecnologias de ensino quando métodos tradicionais fracassam.
\end{abstract}

Palavras-chave: Ensino sem erro, modelagem de estímulos, esvanecimento, dica atrasada.

\section{Errorless Learning and Discrimination Learning}

\section{Abstract}

The term errorless learning is largely used to refer to programming teaching contingencies which result in accurate performances or learning without errors. The occurrence of errors during learning has been considered an important and necessary part of learning in cultural and academic contexts. Errors and their implications for learning are investigated in behavioral analytic studies on stimuli control and their results indicate that errors can affect the accuracy of an already established performance, generate emotional behaviors, and avoidance of the task or teacher. The present study describes different procedures

Endereço para correspondência: Departamento de Processos Psicológicos Básicos, Instituto de Psicologia, Universidade de Brasília, Campus Universitário Darci Ribeiro, Brasília, DF, Brasil 70910-900. E-mail: melo.rm@gmail.com, carmojs@gmail.com e elenicehanna@gmail.com

Os autores são pesquisadores do Instituto Nacional de Ciência e Tecnologia (INCT-ECCE), este apoiado pelo Ministério da Ciência, Tecnologia e Inovação (MCTI) no âmbito do Edital 15/2008, com auxílio do Conselho Nacional de Desenvolvimento Científico e Tecnológico (CNPq; \#573972/2008-7) e da Fundação de Amparo à Pesquisa do Estado de São Paulo (FAPESP; \#2008/57705-8). 
with gradual stimulus change, to teach discrimination skills with none or few errors, and the factors that affect the efficacy of those discriminative training. Such procedures are a relevant contribution from behavior analysis to applied contexts, especially for the development of educational technologies when traditional methods fail.

Keywords: Errorless instruction, stimulus shaping, fading, delayed prompt.

\section{Aprendizaje Sin Error y la Discriminación Aprendizaje}

\section{Resumen}

El término aprendizaje sin error se ha utilizado para referirse, en general, la programación de contingencias de enseñanza que se traducen en rendimientos precisos o con poco errores. La ocurrencia de errores durante el aprendizaje se ha considerado importante y necesario para el aprendizaje en la cultura y en la academia. En el Análisis de la Conducta errores y sus implicaciones para el aprendizaje son investigados en los estudios sobre control de estímulos y los resultados indican que errores puede afectar la precisión de rendimiento ya aprendido, generar comportamientos emocionales, evitación de la tarea o del maestro. En este texto, se presentarán diferentes procedimientos de modificación gradual de estímulos para enseñar la discriminación, que puede generar el aprendizaje sin error y los factores que afectan la efectividad de estas contingencias. Estos procedimientos deben difundirse más ampliamente, constituyen importantes contribuciones para contextos de análisis aplicado del comportamiento, especialmente para el desarrollo de tecnologías educativas cuando fallan los métodos tradicionales.

Palabras clave: Enseñanza sin error, moldeamiento de control de estímulos, desvanecimiento, inductor demorado.

O termo aprendizagem sem erro se refere, de maneira ampla, a um conjunto de procedimentos de ensino que resultam em desempenhos precisos ou com pouco erro. Diferentes perspectivas da psicologia atribuem aos erros uma função positiva no processo de aprendizagem, especialmente no contexto acadêmico. Na Análise do Comportamento os erros e suas implicações para a aprendizagem são investigados em estudos sobre controle de estímulos e os resultados indicam que erros podem interferir na precisão de desempenhos já aprendidos e gerar comportamentos emocionais. Nesse artigo, inicialmente serão revisadas algumas concepções do erro como parte inerente do processo de aprendizagem. Posteriormente, serão caracterizadas programações de contingências de ensino de discriminações que reduzem a ocorrência de erros, fundamentadas em estudos empíricos da Análise do Comportamento, e analisados fatores que favorecem a sua eficácia.

A noção de que a ocorrência de erros é benéfica para a aprendizagem constitui-se em uma explicação culturalmente aceita. A expressão "errando é que se aprende" é comumente mencionada para se referir ao processo de modificação de comportamento inerente à aprendizagem. No meio acadêmico, os erros geralmente são considerados parte do processo de aprendizagem e se constituem em oportunidades para a aquisição de novos comportamentos. Este modo de pensar pressupõe que as respostas incorretas fornecem ao aprendiz "informações" úteis sobre como corrigi-las e emitir o desempenho correto. $\mathrm{O}$ erro, em contexto de avaliação, pode indicar onde o aprendiz se encontra no processo de aprendizagem ou desenvolvimento (La Taille, 1997; Piaget, 1976; Queiroz, Dias, Chagas \& Nepomoceno, 2011).

O interesse pela função dos erros no processo de aquisição de novos comportamentos pode ser evidenciado em estudos de diferentes perspectivas teóricas. As investigações sobre memória da Psicologia Cognitiva ilustram a relação entre os erros e a aquisição de conhecimento. Ebbinghaus (1885/1913) verificou que uma das 
variáveis responsáveis pelo desenvolvimento da habilidade para recordar listas de sílabas sem sentido (e.g., DAX, BUP, LOC) é o número de repetições das listas. As primeiras repetições estavam associadas com mais erros, e à medida que a retenção aumentava, os erros diminuíam gradualmente.

$\mathrm{Na}$ teoria do desenvolvimento cognitivo proposta por Piaget é enfatizada a função dos erros no processo de aquisição de novos conhecimentos, como pode ser observado na seguinte afirmação:

Com efeito, do ponto de vista da invenção um erro corrigido pode ser mais fecundo que um êxito imediato, porque a comparação da hipótese falsa e suas consequências proporciona novos conhecimentos, e a comparação entre erros dá lugar a novas idéias. (Piaget, 1976, pp. 60-61)

De acordo com essa perspectiva, ao se deparar com um problema a criança utiliza esquemas cognitivos que já possui e formula hipóteses a respeito de alternativas de solução e de sequências de ação. Correções na forma de compreender o problema e de como resolvê-lo são gradualmente efetuadas em função dos acertos e dos desequilíbrios na estrutura de conhecimentos gerados pelos erros. Pressupõe-se que ocorreu a construção de novos conhecimentos quando a solução é descoberta e o problema é solucionado (La Taille, 1997; Macedo, 1995; Queiroz et al., 2011). Assim, nessa perspectiva, os erros fazem parte da construção de conhecimentos do aprendiz, são oportunidades para contrastar formas anteriores de compreender um problema e elaborar novas hipóteses, e compete ao professor promover situações que produzam conflitos no sistema cognitivo do aprendiz (Macedo, 1994; Pinto, 2000).

Na perspectiva de precursores da Análise do Comportamento, estudos como os de Thorndike fornecem evidências do interesse pela relação entre a aprendizagem e a ocorrência de erros, da análise da precisão do desempenho em função de mudanças em variáveis ambientais. Segundo a lei do efeito proposta por Thorndike (1911), todos os animais, inclusive o homem, aprendem associações entre estímulo e resposta que são influenciadas pelos resultados ou consequências de satisfação ou de desconforto das respostas. As respostas que geram desconforto podem ser entendidas como erros em vários contextos.

A descrição da aprendizagem em termos de manutenção de respostas que produzem determinados efeitos e diminuição da ocorrência de respostas que não produzem tais resultados, ou erradas, explicitada na Lei do Efeito de Thorndike, está presente em contextos escolares que destacam aspectos da situação que afetam o que o aprendiz é capaz de fazer e explicam a aprendizagem como um processo de aquisição por tentativa-e-erro.

$\mathrm{Na}$ Análise do Comportamento, os erros e suas implicações para a aprendizagem têm sido investigados no âmbito de estudos sobre controle de estímulos, mais especificamente sobre a aquisição de discriminações. O procedimento padrão de treino discriminativo envolve o reforçamento diferencial o qual é frequentemente denominado de tentativa-e-erro. Reforçar diferencialmente implica em apresentar determinada consequência para a ocorrência de uma resposta na presença de um estímulo ( $\mathrm{S}+$ ou $\mathrm{S}^{\mathrm{D}}$ - ésse-dê), e não apresentá-la quando a mesma resposta ocorre na presença de outro(s) estímulo(s) (S- ou $\mathrm{S}^{\Delta}$ - ésse-delta; Bijou, 1995; Catania, 1998/1999; Matos, 1981; Sério, Andery, Gioia \& Micheletto, 2002). Em situações de discriminação, portanto, respostas ao $\mathrm{S}+$ são denominadas de acertos e respostas ao $\mathrm{S}-$, de erros.

A diminuição na ocorrência de erros, ou respostas em S-, é considerada, por alguns autores, como condição necessária para a aprendizagem de discriminações (e.g., Keller \& Schoenfeld, 1950/1974; Spence, 1937). Em uma situação de aprendizagem, inicialmente as respostas são prováveis na presença de diferentes estímulos. Em decorrência do procedimento de reforçamento diferencial, as respostas na presença do $\mathrm{S}+$ (acertos) aumentam de frequência e deixam de ocorrer na presença do S- (erros). O reforçamento de uma resposta, no início do processo, aumenta a probabilidade de ocorrência dessa e de outras respostas na situação presente e na presença de outros estímulos semelhantes. A diferenciação do responder que ocorre no treino discriminati- 
vo é, segundo Keller e Schoenfeld, decorrente da não apresentação do reforço (extinção) como consequência de respostas na presença do S-, reduzindo a probabilidade de ocorrência dessas respostas. Neste sentido, a aquisição de uma discriminação implica em redução da ocorrência de respostas erradas.

Por outro lado, existem resultados de estudos que permitem questionar a importância do erro para a aquisição de discriminações. Diversos estudos mostram que a ocorrência de erros durante o treino discriminativo pode ser influenciada por variáveis de procedimento caracterizadas por alterações nos estímulos antecedentes, como por exemplo, a diferença entre o $\mathrm{S}+\mathrm{e}$ o S- (Frick, 1948; Raben, 1949); a redução gradual da diferença entre S+ e S- (Lawrence, 1952; Schlosberg \& Solomon, 1943); e a quantidade de exposição a tentativas com apenas o $\mathrm{S}+$, antes da apresentação sucessiva do S+e do S- (Skinner, 1938). Considerando as evidências sobre a relação entre os erros e manipulações nos estímulos antecedentes, Terrace (1963) realizou um estudo com pombos, para avaliar o efeito da quantidade de treino das respostas na presença do $\mathrm{S}+$, antes da introdução do S-, e da diferença entre o S+ e S-, na aquisição de discriminação de cor ( $\mathrm{S}+$ : Vermelho; S- : Verde). Foi utilizado um procedimento de treino sucessivo e os sujeitos foram expostos a uma das quatro condições experimentais: (a) Treino Constante com apresentação do $\mathrm{S}+\mathrm{e}$ do S- durante todo treino (tentativa-e-erro); (b) Treino Constante com a apresentação do Sapós algumas sessões de treino com a apresentação apenas do S+; (c) Treino Progressivo com introdução gradual do S- (mudanças progressivas do brilho, duração e cor do S-); (d) Treino Progressivo com introdução gradual do S- após sessões de treino com a apresentação apenas do S+. Nos Treinos Progressivos, denominados de esvanecimento (fading), a duração da apresentação do S- aumentou gradualmente e a intensidade da luz mudou de escuro para verde cada vez mais forte até que diferisse do $\mathrm{S}+$ apenas pela cor. Os treinos progressivos resultaram em poucas respostas na presença do S- e mais erros ocorreram para os sujeitos expostos ao Treino Constante, principalmente quando a cor verde
(S-) era introduzida após sessões de treino com apresentação apenas da cor vermelha (S+). Foi verificado também que uma história de aprendizagem com erros resultou em persistência dos erros e gerou subprodutos emocionais. Terrace concluiu que a aquisição de uma discriminação pode e deve ocorrer sem erros.

Os resultados previamente relatados questionam, portanto, o pressuposto de que os erros são inerentes ao processo de aprendizagem e demonstram que a ocorrência de erros está relacionada com o procedimento de ensino utilizado, ou seja, com o planejamento da contingência de ensino. Adicionalmente, demonstram que os erros podem prejudicar ou retardar a aquisição de discriminações.

O trabalho de Terrace (1963) suscitou investigações adicionais e interesse tanto teórico quanto para a aplicação, sugerindo mudança em antigas concepções de aprendizagem que enfatizam o "erro". Os resultados obtidos por Terrace foram replicados com crianças pré-escolares (Goldiamond \& Moore, 1964; Griffiths \& Griffiths, 1976), crianças autistas (Rincover, 1978; Schreibman \& Charlop, 1981), indivíduos com retardo mental (Sidman \& Stoddard, 1966, 1967; Strand, 1989), e adultos (Baker \& Osgood, 1954; Fields, 1981). No ensino de discriminações têm sido empregados estímulos como: cores, linhas com diferentes inclinações, formas geométricas (e.g., círculo-elipse; círculo-triângulo), tamanho, letras, símbolos Braille, dentre outros.

$\mathrm{Na}$ literatura sobre controle de estímulos, o termo aprendizagem sem erro é utilizado no contexto de procedimentos de modificação gradual de estímulos que resultam em desempenhos precisos, com nenhum ou pouco erro, quando comparado com o procedimento de tentativa-e-erro. McIlvane e Dube (1992) sugerem que todos esses procedimentos modelam o controle de estímulo sendo, por isso, chamados de procedimentos de modelagem de controle de estímulos.

A demonstração de que a aprendizagem pode ocorrer sem erro ou com poucos erros é uma contribuição significativa para a análise das contingências de ensino que geram muitos erros, como é o caso do ensino de matemática (Carmo, 2003), para o desenvolvimento de tecnologias de 
ensino sem erro (Skinner, 1968/1972) e para indivíduos com baixa resistência à frustração, com atraso no desenvolvimento ou que fracassam com os métodos tradicionais (Grow, Carr, Kodak, Jostad \& Kisamore, 2011; Hanney \& Tiger, 2012; Jerome, Frantino \& Sturmey, 2007; Mueller, Palkovic \& Maynard, 2007; Sidman, 2010; Stoddard, de Rose \& Mcllvane, 1986; Stoddard, McIlvane \& de Rose, 1987). Os procedimentos de ensino sem erro geram discriminações em curto espaço de tempo e com menos erros do que os procedimentos tradicionais. Os erros, mesmo ocasionais, podem interferir na precisão de desempenhos já aprendidos, gerar comportamentos de esquiva, tanto da tarefa quanto do professor (e.g., recusas em responder e participar de atividades propostas), e respostas emocionais (e.g., agitação na cadeira, expressões faciais de desagrado; Matos, 1995; Stoddard et al., 1986).

Procedimentos que diminuem a probabilidade de aprendizagem com erros também têm sido associados com melhor desempenho em estudos sobre memória com adultos e crianças (e.g., Anderson \& Craik, 2006; Hammer, Tempelmann \& Münte, 2013; Warmington, Hich \& Gathercole, 2013) e utilizados como estratégias de reabilitação para indivíduos que apresentam déficits de memória e amnésia (e.g., Baddeley \& Wilson, 1994; Clare, Wilson, Carter, Roth \& Hodges, 2002) e com esquizofrenia (Kern et al., 2009). Entretanto, tais estratégias não serão detalhadas uma vez que não envolvem procedimentos de mudança gradual nos estímulos apresentados em tarefas de ensino de discriminações e, portanto, estão além dos objetivos do presente artigo.

No Brasil, entretanto, a divulgação dos procedimentos de ensino de discriminações sem erros é modesta, sendo ainda restrita às pesquisas desenvolvidas no meio acadêmico e predominante na literatura estrangeira, com poucas publicações em português (e.g., Alves, Assis, Kato \& Brino, 2011; Kato \& Maranhão, 2012; Postalli, Almeida, Canovas \& de Souza, 2008; Sidman, 1985; Stoddard et al., 1986; Stoddard et al., 1987). A repercussão de possíveis aplicações, mesmo de trabalhos de qualidade como o de Iñesta (1972/1980), que apresenta sugestões claras e sistemáticas para o ensino de de- sempenhos relevantes para as séries iniciais do ensino fundamental, tais como leitura, escrita e operações matemáticas, é inexpressiva. Torna-se relevante, portanto, ampliar a divulgação dos diferentes procedimentos de ensino sem erro e dos fatores relevantes para a efetividade de tais treinos discriminativos.

\section{Procedimentos de Ensino sem Erro}

Uma análise da literatura sobre controle de estímulos mostra que procedimentos de aprendizagem sem erro podem ser utilizados para ensinar discriminações simples ou condicionais. A essência dos primeiros procedimentos propostos como contingências de ensino sem erro estava na mudança gradual em dimensões dos estímulos antecedentes, sendo utilizado como termo genérico procedimentos de esvanecimento. Atualmente se sabe que outros procedimentos que não incluem a modificação gradual dos estímulos (e.g., exclusão) podem também evitar a ocorrência de erros.

Procedimentos de ensino sem erro apresentam como principais características: (a) modificação gradual em dimensões dos estímulos (e.g., cor, tamanho, forma, brilho) utilizados no treino discriminativo, podendo ocorrer no $\mathrm{S}+$, no $\mathrm{S}-\mathrm{ou}$ no S+ e no S-; e (b) redução das diferenças entre os estímulos das discriminações inicial e final. No início do treino discriminativo geralmente são utilizados estímulos que já controlam o responder diferencial. Ao longo das etapas de ensino são efetuadas modificações, de forma gradual, em uma ou mais dimensões de pelo menos um desses estímulos. Como resultado, poderia haver mudança no controle de estímulo das dimensões inicias para as finais do $\mathrm{S}+\mathrm{e}$ do $\mathrm{S}-\mathrm{com}$ a ocorrência de uma quantidade mínima de erros ao longo do treino discriminativo.

Alguns aspectos devem ser observados durante a etapa de planejamento de uma contingência de ensino sem erro. As manipulações nos estímulos devem ser definidas previamente pelo instrutor (experimentador ou terapeuta) a partir da verificação do que o aprendiz é capaz de fazer, da identificação dos estímulos que já controlam o seu comportamento, de reforçado- 
res potenciais e da constatação de que o procedimento mais simples de reforçamento diferencial foi ineficaz (Eckert \& Browder, 1997; Etzel \& LeBlanc, 1979). A escolha do procedimento deve ser feita considerando-se as características individuais do aprendiz, e o programa deve ser cuidadosamente planejado no que se refere à seleção dos estímulos iniciais e finais, a definição do número de passos de mudança gradual e do critério de aprendizagem a ser adotado.

A literatura descreve pelo menos três procedimentos de ensino sem erro que serão apresentados e analisados neste artigo: (a) esvanecimento; (b) modelagem de estímulo; e (b) dica atrasada.

Esvanecimento (fading). O esvanecimento caracteriza-se pela mudança gradativa em uma ou mais dimensões físicas (e.g., intensidade, tamanho, cor) de pelo menos um dos estímulos discriminativos (S+, S- ou S+ e S-). Inicia-se o treino com uma tarefa de discriminação que o aprendiz já domina, sendo apresentados estímulos que se diferenciam em mais de uma dimensão, e é comum a apresentação apenas do $\mathrm{S}+$ na primeira tentativa de treino, a fim de aumentar a probabilidade de respostas corretas (e.g., Gollin \& Savoy, 1968; Sidman \& Stoddard, 1966; Terrace, 1963). Em etapas sucessivas, modificações graduais ao longo de um contínuo são efetuadas em pelo menos um dos estímulos iniciais até que sejam apresentados os estímulos da discriminação final (ou critério), previamente definida como a que deverá ser ensinada. A diferença entre uma ou mais dimensões do $\mathrm{S}+$ e do $\mathrm{S}$ - é progressivamente reduzida.

A forma mais comum de esvanecimento envolve a mudança gradual na dimensão intensidade (e.g., Gollin \& Savoy, 1968; Sidman \& Stoddard, 1967). Um dos estímulos (S+ ou S-) permanece constante durante todo o treino enquanto o outro é gradualmente introduzido, aumentando-se progressivamente a sua intensidade até se tornar igual à do estímulo mantido constante. A Figura 1 (Painel a) apresenta cinco etapas de uma sequência de mudança gradual efetuada no S- de um treino discriminativo entre círculo (S+) e triângulo (S-). Na tentativa inicial, é apresentado apenas o $\mathrm{S}+$ com as dimensões na for- ma final (e.g., intensidade, forma e tamanho) e o outro estímulo (S-) está ausente (e.g., apresentação de um cartão em branco). Progressivamente, a intensidade do triângulo (S-) aumenta até se igualar ao $\mathrm{S}+$ na etapa final. Tal procedimento tem sido denominado de fading in, esvanecimento aditivo ou introdução gradual de estímulo.

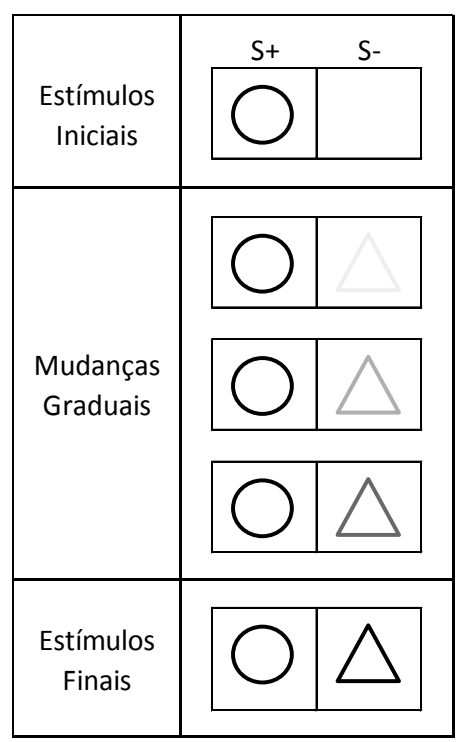

(a)

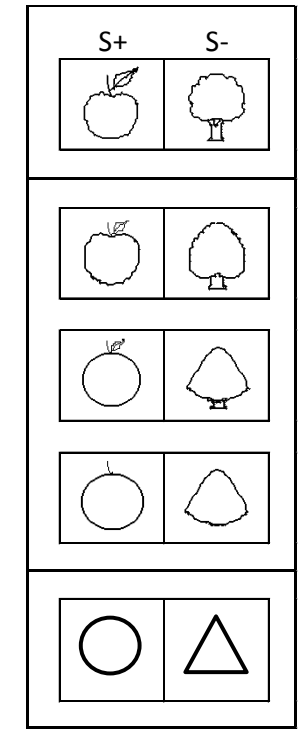

(b)
Figura 1. Exemplo de pares de estímulos de um procedimento de esvanecimento (Painel a) e do procedimento de modelagem de estímulos (Painel b).

O procedimento de esvanecimento pode incluir a introdução ou retirada de estímulos que já controlam o responder discriminado (chamados de dica ou prompt) aos estímulos da discriminação final (Lancioni \& Smeets, 1986). A dica se refere tanto a um estímulo adicionado, como à apresentação de uma dimensão do estímulo, que não é a alvo da discriminação, de maneira ressaltada ou exagerada. A dica é um estímulo suplementar não diretamente relacionado com a tarefa, mas inserido nela porque já controla o desempenho a ser ensinado (Touchette \& Howard, 1984). A sobreposição por si só não se caracteriza como um procedimento de ensino sem erro. Em associação com o esvanecimento, no entanto, ilustra uma de suas variações, a remoção gradual de estímulo, procedimento denominado de fading out.

Iñesta (1972/1980) apresenta diversos exemplos desses procedimentos para treinar re- 
pertórios verbais em crianças com atraso no desenvolvimento. Em um dos exemplos, o ensino de responder diferencial às palavras impressas bolo e bola é iniciado com a apresentação apenas das figuras, considerando-se que as crianças prontamente nomeiam as figuras. Ao longo das tentativas sucessivas, gradativamente os estímulos finais são introduzidos (palavras impressas: bolo e bola). A introdução das letras, uma a uma, pode ser efetuada aumentando-se gradualmente a intensidade das linhas, iniciando com linhas pontilhadas, passando para tracejadas e finalmente contínuas. Nesta etapa intermediária, a nomeação ocorre na presença do estímulo composto palavra impressa - figura. A seguir, inicia-se a remoção gradual da figura. Na situação final, a criança estará dizendo "bolo" diante da palavra bolo e "bola" diante de bola tendo sido, portanto, modificado o controle dos estímulos iniciais (figuras) para os estímulos finais (palavras impressas).

Modelagem de Estímulo (stimulus shaping). A modelagem de estímulo se refere ao procedimento que modifica gradualmente a topografia (configuração ou forma) do(s) estímulo(s) inicial(is) até que eles adquiram a forma dos estímulos da discriminação final (Etzel \& LeBlanc, 1979; Schilmoeller, Schilmoeller, Etzel \& LeBlanc, 1979). O treino inicia-se com estímulos fisicamente distintos e que já controlam desempenho discriminado, sendo geralmente utilizados estímulos familiares; manipulações progressivas são efetuadas na forma dos estímulos iniciais, de tal maneira que ao término das etapas de mudança os estímulos apresentam a forma dos estímulos da discriminação final, previamente determinados. Os estímulos iniciais e finais compartilham uma mesma dimensão crítica que permite a diferenciação entre o $\mathrm{S}+\mathrm{e} o \mathrm{~S}-$.

Em um exemplo de procedimento para ensinar a discriminação entre círculo $(\mathrm{S}+$ ) e triângulo (S-), a modelagem de estímulo inicia-se com a apresentação de estímulos com formas distintas tais como uma maçã ( $\mathrm{S}+$ ) e uma árvore (S-; Figura 1, Painel b). A configuração da árvore é modificada gradualmente até a forma de um triângulo, enquanto que a forma da maçã é mudada progressivamente para a forma de um círculo.
$\mathrm{Na}$ tentativa final são apresentados o $\mathrm{S}+\mathrm{e}$ o Sda discriminação final, o círculo e o triângulo.

De forma semelhante ao esvanecimento, a modelagem de estímulo pode ser combinada com a sobreposição de uma dica. Etzel, LeBlanc, Schilmoeller e Stella (1981) utilizaram a denominação sobreposição e modelagem de estímulo para se referir à situação em que a modelagem é efetuada no elemento que é adicionado ao estímulo da discriminação final, para diferenciar esse procedimento da modelagem de estímulo em que o estímulo inicial é transformado no estímulo complexo final a partir de mudanças na sua configuração. No procedimento descrito por Etzel et al. (1981) foram acrescentados aos estímulos da discriminação final (figuras que se diferenciavam pelo ângulo de inclinação entre linhas, $\Lambda \mathrm{e} /$ ) dois elementos, um cachorro conectado por uma linha à figura com ângulo $\Lambda(\mathrm{S}+)$ e um balão conectado por uma linha à figura com ângulo / (S-). Ao longo das etapas de mudança dos elementos adicionados, o balão se desprendia e se deslocava para cima até desaparecer e a forma do cachorro se modificava para representá-lo como se estivesse cavando um buraco, colocando a cabeça e a seguir partes cada vez maiores do corpo até entrar completamente no buraco e desaparecer. Na sobreposição e modelagem de estímulos, portanto, são sobrepostos aos estímulos da discriminação final ( $\mathrm{S}+$ e $\mathrm{S}-$ ou apenas $\mathrm{S}+$ ou $\mathrm{S}-$ ) estímulos adicionais que já controlam responder diferencial. A topografia dos estímulos adicionados é progressivamente modificada até serem incorporados aos estímulos finais ou removidos por partes.

A escolha entre a utilização do esvanecimento ou da modelagem de estímulos requer uma análise cuidadosa da discriminação a ser ensinada. Estudos anteriores que compararam os dois procedimentos relataram que a modelagem de estímulo gera a aprendizagem de discriminações mais precisas quando a discriminação alvo envolve a forma dos estímulos. Dificuldade de ensino de discriminações com o procedimento de esvanecimento está relacionada ao fato de que, em geral, a dimensão manipulada ao longo das etapas do procedimento é diferente daquela definida como critério da discriminação final (Etzel \& LeBlanc, 1979; Schilmoeller et 
al., 1979). Esta distinção entre dimensão manipulada e dimensão da discriminação final pode ser verificada analisando-se os exemplos apresentados nos painéis a e $b$ da Figura 1 . No esvanecimento (Painel a) a dimensão modificada é a intensidade da linha de contorno das figuras. As discriminações iniciais, por isso, podem ser feitas com base na diferença da intensidade das linhas, enquanto que a discriminação final, que deve ser aprendida, envolve a forma dos estímulos. Na modelagem de estímulo (Painel b), a dimensão modificada desde o início é a forma dos estímulos, passando do formato de maçã e arvore para formas cada vez mais próximas às formas de círculo e triângulo, que são os estímulos finais. O sucesso no ensino de discriminações com esses procedimentos depende, portanto, de um planejamento de contingência que desde o início focalize o controle pela dimensão relevante, partindo-se de estímulos que o aprendiz já seja capaz de discriminar. Outras variáveis importantes serão discutidas posteriormente.

Dica Atrasada (delayed prompting). O procedimento de dica atrasada foi desenvolvido por Touchette (1971) e se refere à modificação gradual do intervalo entre a apresentação dos estímulos da discriminação final e da apresentação da dica se a resposta não ocorrer. Inicialmente, a dica é sobreposta (apresentada simultaneamente) a um dos estímulos da discriminação final ( $\mathrm{S}+$ ou S-) e, ao longo das etapas de ensino, interpõe-se um tempo para que a resposta ocorra entre a apresentação dos estímulos finais e da dica. Este tempo é gradualmente aumentado até que a dica possa ser removida definitivamente, quando as respostas passam a ocorrer antes da sua apresentação (Graff \& Green, 2004; Touchette \& Howard, 1984). São frequentemente utilizados como dica por um professor, por exemplo, o apontar a alternativa correta, a colocação de uma cor, figura, instruções verbais, gestos, ajuda física ou o modelo da resposta a ser emitida.

Seria um procedimento de dica atrasada no ensino da discriminação círculo-triângulo, a apresentação dos dois estímulos finais e imediatamente (após 0,1 s) o S- desaparece do cenário, ficando apenas o círculo que deve ser escolhi- do. Após um pequeno intervalo (importante para separar as tentativas), apresentam-se novamente os estímulos finais, agora atrasando a retirada do triângulo por um tempo um pouco maior $(0,5 \mathrm{~s}$ por exemplo) e novamente, se a resposta não ocorrer durante esse intervalo, retira-se o S- do cenário.

Em um segundo exemplo deste procedimento, pode-se utilizar como dica, ao ensinar discriminações entre orientações diferentes de formas geométricas $(\triangle-\mathrm{S}+\mathrm{e} \nabla-\mathrm{S}-)$, uma seta na cor vermelha $(\boldsymbol{\nabla})$ para indicar a escolha correta. Os estímulos da discriminação final são apresentados ao longo de todo o treino e a dica aparece, inicialmente, junto com os outros estímulos. A escolha do $\mathrm{S}+$ em uma tentativa resulta em reforçamento e no aumento do atraso entre a apresentação dos estímulos correto e incorreto e da dica. Erros produzem a diminuição do atraso. Ao longo das tentativas, os atrasos aumentam gradualmente, até que a seta seja completamente removida.

Procedimentos de ensino sem erro são especialmente úteis para crianças com dificuldade de aprendizagem, quando erros recorrentes acontecem. Os procedimentos têm se mostrado úteis para ensinar discriminações entre estímulos gráficos (e.g., Griffiths \& Griffiths, 1976; Melo, de Jesus \& Hanna, 2005; Schilmoeller et al., 1979), que é uma habilidade relevante para a aprendizagem de desempenhos mais complexos que requerem diferenciação de formas, tais como ler e escrever. Em uma revisão de 30 estudos empíricos que utilizaram a dica atrasada, Browder, Ahlgrim-Delzell, Spooner, Mims e Baker (2009) verificaram a efetividade desse procedimento para ensinar reconhecimento de palavras e figuras para indivíduos com atraso no desenvolvimento e recomendaram a sua aplicação em contextos escolares.

A dica atrasada tem sido utilizada com sucesso no ensino do comportamento de seguir instruções, que é um desempenho relevante no contexto acadêmico e clínico. Striefel, Wetherby e Karlan (1979), por exemplo, ensinaram respostas motoras por meio de imitação a adolescentes com deficiência intelectual. Posteriormente, a 
instrução verbal (verbo de ação + substantivo) era apresentada simultaneamente com o modelo da resposta motora (dica) executada pelo experimentador. Respostas corretas resultavam, na próxima tentativa, em atrasos cada vez maiores entre a instrução verbal e o modelo apresentado ao aprendiz. Ao longo do programa foi verificada mudança no controle de estímulo do modelo executado pelo experimentador para as instruções verbais.

Procedimentos para modelar o controle de estímulos são frequentemente utilizados na confecção de quadros de uma instrução programada, com o objetivo de aumentar a probabilidade de acertos. Os quadros apresentam pequenas quantidades de informação e são organizados em uma sequência que avança do mais simples para o mais complexo. $\mathrm{O}$ aprendiz deve emitir pelo menos uma resposta por quadro. Dicas ou informações adicionais que já controlam o responder diferencial ou que "indicam" a resposta a ser emitida são acrescentadas e, em seguida, esvanecidas (Sidman, 2010; Skinner, 1968/1972; Tabler, Glaser \& Schaefer, 1965). Por exemplo, figuras que indicam a palavra que deverá ser escrita (e.g., $\theta, \varangle$ ), a parte inicial da palavra (e.g., $\left.d e_{-}\right)$, ou um termo ou frase com significado similar (e.g., Uma dezena de bananas é outro nome para _ bananas). Ao longo da sequência de quadros a dica é removida e as respostas passam a ocorrer diante de novas situações ou estímulos discriminativos.

\section{Fatores que Afetam a Eficácia de Treinos Discriminativos}

$\mathrm{Na}$ literatura sobre controle de estímulos, verifica-se que os estudos que utilizam procedimentos de ensino sem erro, em geral, envolvem participantes com atraso no desenvolvimento, tarefas de discriminação simultânea entre estímulos visuais e, geralmente, enfatizam a comparação entre procedimentos distintos. No entanto, deve-se levar em consideração que vários fatores afetam a eficácia de qualquer treino discriminativo. Entre esses fatores estão: (a) o tipo de treino (sucessivo - com as alternativas de escolha apresentadas separadamente; simultâneo - com as opções correta e incorretas presentes ao mesmo tempo), (b) características dos participantes (idade, história passada, nível de desenvolvimento), (c) relação entre a(s) dimensão(ões) em que os estímulos $\mathrm{S}+$ e S- se diferenciam e a dimensão da discriminação alvo (mesma dimensão ou dimensões diferentes), (d) qualidade física dos estímulos (e.g., nitidez), (e) topografia da resposta (e.g., apontar, nomear), (f) esquema de reforçamento utilizado, (g) tipo de reforço e parâmetros (magnitude, frequência e atraso) do reforçamento (Harrison, 1991). Adicionalmente, é importante saber quais condições já foram identificadas como importantes para que os procedimentos de ensino sem erro sejam eficazes.

A dimensão do estímulo modificada tem sido apontada como um dos principais fatores que afetam a precisão da aquisição de discriminações (Schilmoeller et al., 1979; Strand \& Morris, 1988). Dimensões relacionadas com o critério se referem àquelas dimensões críticas para a discriminação final, enquanto que dimensões não relacionadas com o critério envolvem modificações em uma dimensão irrelevante para a discriminação final (Etzel \& LeBlanc, 1979). Ao apresentar a comparação entre esvanecimento e modelagem de estímulos, já apresentamos exemplo sobre esta questão.

Os termos manipulação intra-estímulo vs extra-estímulo têm sido também utilizados (Schreibman, 1975) para indicar a dimensão dos estímulos antecedentes modificada. Em uma tarefa de discriminação das letras E (S+) e F (S-), por exemplo, apresentar na primeira tentativa a letra $\mathrm{E}$ com o traço inferior maior e mais largo e, gradualmente reduzir estas dimensões é uma manipulação intra-estímulo. A sobreposição das letras em fundos de cores diferentes, vermelho (E) e verde (F), seguida de esvanecimento das cores, ilustra uma manipulação extra-estímulo. Os resultados de estudos que investigaram tais manipulações mostram que, em geral, mudanças graduais em dimensões relacionadas com o critério (Schilmoeller et al., 1979; Strand \& Morris, 1988) e de dimensões intra-estímulo (Rincover, 1978; Schreibman, 1975) produziram discriminações mais precisas. A eficácia deste procedimento é atribuída ao fato de que a dimensão dos 
estímulos iniciais manipulada é também a base para a discriminação final, mantendo-se ao longo do treino o controle de estímulo por uma mesma dimensão.

A manipulação de dimensões não relacionadas com o critério e de dicas extra-estímulo tem gerado erros nas etapas finais da aprendizagem de discriminações com procedimento de ensino "sem erro" (Koegel \& Rincover, 1976; Schwartz, Firestone \& Terry, 1971). Quando tais manipulações são efetuadas, tende a ser estabelecido o controle pela dimensão manipulada ou pela dica adicionada aos estímulos iniciais. Ao longo das etapas de mudança gradual, a dica é gradualmente removida e a diferença entre os estímulos finais sem a dica pode ser ineficaz como base para a discriminação. A ocorrência de respostas incorretas nesse estágio mostra que a transferência de controle de uma dimensão do estímulo para outra não ocorreu.

Nos procedimentos de ensino sem erro podem ser efetuadas modificações nas dimensões dos estímulos definidos como S+ ou S-, ou dos dois estímulos ( $\mathrm{S}+$ e $\mathrm{S}-$ ), simultaneamente ou sucessivamente. $\mathrm{O}$ estímulo em relação ao qual serão efetuadas as modificações graduais é outra variável que pode afetar o desempenho durante o processo de aprendizagem de uma discriminação. Os resultados de estudos que avaliaram o efeito da modificação gradual nos diferentes estímulos são variados entre os estudos, mas a maioria relata efeitos sistemáticos. Schreibman e Charlop (1981) e Stella e Etzel (1986) observaram que a modificação do $\mathrm{S}+$ resultou na aprendizagem de discriminações mais rápidas e com menos erros em comparação com a modificação do S- (ver também Fields, 1978). Cheney e Stein (1974) e Strand (1989) verificaram resultados semelhantes nos treinos com modificação do S+ ou do S- (ver também Zawlocki \& Walls, 1983). Estudos adicionais são necessários, portanto, para possibilitar conclusões sobre os efeitos dessa variável.

Estudos que manipularam a ordem em que as modificações são efetuadas em $\mathrm{S}+\mathrm{e}$ em S- sugerem que há uma relação entre esta variável e os desempenhos nos treinos. Nos estudos que efetuaram modificações sucessivas dos estímu- los $\mathrm{S}+$ e S-, a modificação do $\mathrm{S}+$ por último tem sido mais utilizada com a argumentação de que tal procedimento resulta em controle pelo $\mathrm{S}+\mathrm{e}$ ausência ou poucos erros no treino (e.g., McCartney \& LeBlanc, 1997; Schilmoeller et al., 1979). Essa sugestão tem sido apoiada em resultados de estudos que investigaram o movimento dos olhos durante a aquisição de discriminações com modificações graduais do $\mathrm{S}+$ e do S-, os quais ressaltam que o organismo tende a olhar em direção aos estímulos que estão sendo modificados (Stella \& Etzel, 1986).

\section{Ensino Com e Sem Erro?}

Em geral, estímulos utilizados em treinos discriminativos possuem diversas dimensões (cor, forma, tamanho, número de traços, etc.). Quando uma discriminação é adquirida a partir de um treino discriminativo, não é trivial a pergunta "O que foi aprendido?" O responder diferencial ocorre apenas aos estímulos treinados? Se sim, seria correto, por exemplo, dizer que houve aprendizagem da discriminação de cor? $\mathrm{Ou}$ aprendeu-se a discriminar aqueles estímulos coloridos? Estudos em que procedimentos de ensino sem erro são utilizados, em geral, não incluem testes para avaliar a extensão do controle de estímulo estabelecido no treino. Dentre os poucos experimentos que avaliaram esta questão e realizando testes, Aeschleman e Higgins (1982) verificaram que treinos com esvanecimento produziram desempenhos consistentes em testes de generalização com novos exemplares dos estímulos (garrafas e jarras), sugerindo a aprendizagem de conceitos. Resultados semelhantes foram relatados por Van Laarhoven, Johnson, Repp, Karsh e Lenz (2003) ensinando discriminações mais complexas (condicionais). Por outro lado, Melo et al. (2005) mostraram que o uso de modelagem de estímulos e esvanecimento para treinar discriminações de posição (dentro, em cima, esquerda) resultou em desempenhos próximos ao acaso em testes diante de estímulos novos. Desempenhos melhores nos testes foram obtidos com o procedimento de tentativa-e-erro, o que levou os autores a sugerir que, desempenhos consistentes nos testes de generalização após o 
ensino com procedimento sem erro, pode depender da inclusão de um bloco de tentativas com os $\mathrm{S}+$ e S-, após a etapa de mudança gradual. A combinação de procedimentos, com mudança gradual seguido de tentativa-e-erro, parece necessária para a aquisição das discriminações, previne subprodutos dos erros e gera controle de estímulos pelas dimensões relevantes.

No ensino de discriminações, o estabelecimento de controle por parte ou dimensão muito específica do estímulo treinado é chamado de controle seletivo de estímulos (Allen \& Fuqua, 1985) ou controle restrito (e.g., Dube \& McIlvane, 1997) ou superseletividade (e.g., Lovaas \& Schreibman, 1971). Este controle é identificado quando posteriormente são efetuadas mudanças nas contingências de reforçamento ou apresentadas tarefas mais complexas de teste que demandam que o aprendiz responda com base em mais de uma dimensão ou em uma dimensão diferente dos estímulos (Pierce \& Epling, 1995; Stromer, 1979). Strand (1989) observou que, após o treino discriminativo inicial, quando a função dos estímulos $\mathrm{S}+\mathrm{e} \mathrm{S}$ - foi invertida (reversão da discriminação), crianças com deficiência intelectual tendiam a continuar respondendo ao tamanho exagerado de um elemento do $\mathrm{S}+$ (dica) do treino original. Gollin e Savoy (1968) verificaram, após o treino de duas discriminações de forma com esvanecimento, que os desempenhos precisos nos treinos não foram mantidos quando os dois pares $\mathrm{S}+$ - S- eram apresentados randomicamente em um teste. Ao replicarem o estudo de Gollin e Savoy, Schilmoeller et al. (1979) verificaram desempenhos mais precisos nos testes quando o controle superseletivo foi reduzido por um treino que utilizava modelagem de estímulos. Tais resultados sugerem que o controle por dimensões irrelevantes pode ser estabelecido durante as etapas de mudanças graduais que utilizam dimensões ou dicas irrelevantes para as discriminações finais ao longo dos treinos com esvanecimento. Sobre este assunto, existe uma extensa literatura relacionando superseletividade com dificuldade de aprendizagem em crianças com limitação cognitiva e autistas (e.g., Allen \& Fuqua, 1985; Dittlinger \& Lerman, 2011; Lovaas, Koegel \& Schreibman, 1979).

\section{Considerações Finais}

O conjunto de estudos com os procedimentos e resultados apresentados neste trabalho mostram evidências de que o erro não é necessário para a aprendizagem. Quando este é minimizado, evita-se também os seus subprodutos emocionais. Entretanto, a ocorrência de erros pode, em situação de avaliação, indicar controles por dimensões irrelevantes dos estímulos e auxiliar no planejamento instrucional individual. Nesse sentido, identificar as fontes ambientais geradoras de desempenhos inadequados é fundamental no planejamento, e também auxilia na superação de uma concepção amplamente difundida no meio acadêmico de que a responsabilidade pelo sucesso ou fracasso recai exclusivamente sobre o aluno.

Este artigo representa uma contribuição para a maior difusão deste conhecimento no Brasil, tendo em vista a possibilidade de utilização dos procedimentos que reduzem a probabilidade de respostas incorretas, em diferentes contextos de aplicação, para ensinar: discriminações envolvidas em atividades de leitura, como na discriminação de letras com forma semelhante ou fontes diferentes (e.g, Smeets \& Striefel, 1990; Van Laarhoven et al., 2003); habilidades matemáticas para crianças com atraso no desenvolvimento (e.g., Burton, Anderson, Prater \& Dyches, 2013); habilidades comunicativas para crianças autistas (e.g., Ostryn \& Wolfe, 2011; Raulston et al., 2013); no tratamento para esquiva social (e.g., Harper, Iwata \& Camp, 2013), para citar alguns. É importante salientar que o uso de procedimentos especiais que demandam um planejamento especial é justificado quando erros são recorrentes ou para acelerar o ensino de habilidades complexas que apresentam muitos erros na fase inicial de aquisição.

O desenvolvimento de tecnologias de ensino que visem a redução dos erros e seus efeitos sobre o desempenho do aprendiz exige, no entanto, planejamento sistemático, treinamento de recursos humanos, elaboração de material especializado, disponibilidade de tempo para aplicação e avaliação constante. A aplicação desta tecnologia poderia assessorar o planejamento e 
a elaboração de material didático, sobretudo nas séries iniciais e no ensino especial, nas quais se observa frequentemente dificuldade na aprendizagem de determinadas discriminações visuais e auditivas, como por exemplo, a diferenciação entre $\mathrm{p} / \mathrm{q}, \mathrm{d} / \mathrm{b}$ e f/v, $\mathrm{t} / \mathrm{d}$ ou dos símbolos matemáticos das operações aritméticas $+/-,+/ \times,+/ \div,=/ \neq$, $\leq \geq$. A possibilidade de prevenir tais dificuldades a partir dessas e outras novas metodologias de ensino pode significar a prevenção ou redução de dificuldades na alfabetização e do fracasso escolar no ensino regular ou especial.

O psicólogo escolar, apoiado nos conhecimentos advindos de pesquisas básicas e aplicadas sobre aprendizagem sem erro, ao lidar com as diferentes relações presentes nas situações de ensino e de aprendizagem, pode fornecer importantes elementos à mudança de práticas de ensino, como: superação da visão de que erros são necessários à aprendizagem; retirada do foco de responsabilidade no aluno, com indicação das fontes ambientais geradoras de erro; auxílio na programação de etapas graduais de ensino com o objetivo de garantir controle efetivo de respostas adequadas; ampliação das possibilidades de planejamento eficaz dos professores; ampliação das possibilidades de uma avaliação da aprendizagem a partir da análise da efetividade da programação de ensino; prevenção de respostas emocionais geradas e mantidas pela ocorrência de erros; aumento da motivação de professores e alunos a partir de programações de ensino que possibilitam o sucesso ao invés do fracasso na aprendizagem. Em suma, os estudos sobre aprendizagem sem erro contribuem, sobremaneira, à mudança de foco no trabalho, tanto do psicólogo escolar quanto dos demais profissionais da educação, no sentido de centrarem seus esforços na produção do sucesso dos alunos.

\section{Referências}

Aeschleman, S. R., \& Higgins, A. F. (1982). Concept learning by retarded children: A comparison of three discrimination learning procedures. Journal of Mental Deficiency Research, 26, 229-238.

Allen, K. D., \& Fuqua, R. W. (1985). Eliminating selective stimulus control: A comparison of two procedures for teaching mentally retarded children to respond to compound stimuli. Journal of the Experimental Child Psychology, 39, 55-71.

Alves, K. R. S., Assis, G. J. A., Kato, O. M., \& Brino, A. L. F. (2011). Leitura recombinativa após procedimentos de fading in de sílabas das palavras de ensino em pessoas com atraso no desenvolvimento cognitivo. Acta Comportamentalia, 19, 183-203.

Anderson, N. D., \& Craik, F. I. M. (2006). The mnemonic mechanisms of errorless learning. Neuropsychologia, 44, 2806-2813.

Baddeley, A., \& Wilson, B. A. (1994). When implicit learning fails: Amnesia and the problem of error elimination. Neuropsychologia, 32, 53-68.

Baker, R. A., \& Osgood, S. W. (1954). Discrimination transfer along a pitch continuum. Journal of Experimental Psychology, 48, 241-246.

Bijou, S. W. (1995). Behavior analysis of child development. Reno, NV: Context Press.

Browder, D., Ahlgrim-Delzell, L., Spooner, F., Mims, P. J., \& Baker, J. N. (2009). Using time delay to teach literacy to students with severe developmental disabilities. Exceptional Children, 75, 343-364.

Burton, C. E., Anderson, D. H., Prater, M. A., \& Dyches, T. T. (2013). Video self-modeling on an iPad to teach functional math skills to adolescents with autism and intellectual disability. Focus on Autism and Other Developmental Disabilities, 28, 67-77.

Carmo, J. S. (2003). Dificuldades de aprendizagem ou dificuldade de ensino? Algumas contribuições da Análise do Comportamento. In M. Z. S. Brandão (Ed.), Sobre comportamento e cognição: A história e os avanços, a seleção por contingências em ação (pp. 396-401). Santo André, SP: ESETec

Catania, A. C. (1999). Aprendizagem: Comportamento, linguagem e cognição (4. ed., D. G. Souza, Trad.). Porto Alegre, RS: Artmed. (Original publicado em 1998)

Cheney, T., \& Stein, N. (1974). Fading procedures and oddity learning in kindergarten children. Journal of Experimental Child Psychology, 17, 313-321.

Clare, L., Wilson, B. A., Carter, G., Roth, I., \& Hodges, J. R. (2002). Relearning face-name associations in early Alzheimer's disease. Neuropsychology, 16, 538-547. 
Dittlinger, L. H., \& Lerman, D. C. (2011). Further analysis of picture interference when teaching word recognition to children with autism. Journal of Applied Behavior Analysis, 44, 341-349.

Dube, W. V., \& McIlvane, W. J. (1997). Reinforcer frequency and restricted stimulus control. Journal of the Experimental Analysis of Behavior, 68, 303-316.

Ebbinghaus, H. (1913). Memory: A contribution to experimental psychology (A. R. Ruger \& C. E. Bussenius, Trans.). New York: Teachers College, Columbia University. (Original work published 1885). Retrieved from http://psychclassics. yorku.ca/Ebbinghaus/index.htm

Eckert, T. L., \& Browder, D. M. (1997). Stimulus manipulation: Enhancing materials for self-directed learning. In D. M. Baer \& E. M. Pinkston (Eds.), Environment and Behavior (pp. 80-96). Boulder, CO: Westview Press.

Etzel, B. C., \& LeBlanc, J. M. (1979). The simplest treatment alternative: The law of parsimony applied to choosing appropriate instructional control and errorless-learning procedures for the difficult-to-teach child. Journal of Autism and Developmental Disorders, 9, 361-382.

Etzel, B. C., LeBlanc, J. M., Schilmoeller, K. J., \& Stella, M. E. (1981). Stimulus control procedure in education of young children. In S. W. Bijou \& R. Ruiz (Eds.), Contributions of behavior modification to education (pp. 3-37). Hillsdale, NJ: Lawrence Erlbaum.

Fields, L. (1978). Fading and errorless transfer in successive discriminations. Journal of the Experimental Analysis of Behavior, 30, 123-128.

Fields, L. (1981). Early and late introduction of probes and stimulus control acquisition in fading. Journal of the Experimental Analysis of Behavior, 36, 363-370.

Frick, F. C. (1948). An analysis of an operant discrimination. The Journal of Psychology, 26, 93123.

Goldiamond, I., \& Moore, R. (1964). Errorless establishment of visual discrimination using fading procedures. Journal of the Experimental Analysis of Behavior, 7, 269-272.

Gollin, E. S., \& Savoy, P. (1968). Fading procedures and conditional discrimination in children. Journal of the Experimental Analysis of Behavior, $11,443-451$.
Graff, R. B., \& Green, G. (2004). Two methods for teaching simple visual discriminations to learners with severe disabilities. Research in Developmental Disabilities, 25, 295-307.

Griffiths, K., \& Griffiths, R. (1976). Errorless establishment of letter discriminations with a stimulus fading procedure in pre-school children. Perceptual and Motor Skills, 42, 387-396.

Grow, L. L., Carr, J. E., Kodak, T. M., Jostad, C. M., \& Kisamore, A. N. (2011). A comparison of methods for teaching receptive labeling to children with autism spectrum disorders. Journal of Applied Behavior Analysis, 44, 475-498.

Hammer, A., Tempelmann, C., \& Münte, T. F. (2013). Recognition of face-name associations after errorless and errorful learning: an fMRI study. BMC Neuroscience, 14(1), 30.

Hanney, N. M., \& Tiger, J. H. (2012). Teaching coin discrimination to children with visual impairments. Journal of Applied Behavior Analysis, 45, 167-172.

Harper, J. M., Iwata, B. A., \& Camp, E. M. (2013). Assessment and treatment of social avoidance. Journal of Applied Behavior Analysis, 46, 147160.

Harrison, J. M. (1991). Stimulus control. In I. H. Iversen \& K. A. Lattal (Eds.), Experimental Analysis of Behavior: Part I (pp. 251-299). Amsterdam, Netherlands: Elsevier.

Iñesta, R. E. (1980). Técnicas de modificação do comportamento: Aplicação ao atraso no desenvolvimento (D. P. Soares, Trad.). São Paulo, SP: EPU. (Original publicado em 1972)

Jerome, J., Frantino, E. P., \& Sturmey, P. (2007). The effects of errorless learning and backward chaining on the acquisition of internet skills in adults with developmental disabilities. Journal of Applied Behavior Analysis, 40, 185-189.

Kato, O. M., \& Maranhão, C. M. A. (2012). Procedimentos de ensino de leitura e aprendizagem sem erros. In J. S. Carmo \& M. J. F. X. Ribeiro (Eds.), Contribuições da análise do comportamento à prática educacional (pp. 153-179). Santo André, SP: ESETec.

Keller, F. S., \& Schoenfeld, W. N. (1974). Princípios de Psicologia: Um texto sistemático na ciência do comportamento (C. M. Bori \& R. Azzi, Trads.). São Paulo, SP: EPU. (Original publicado em 1950) 
Kern, R. S., Liberman, R. P., Becker, D. R., Drake, R. E., Sugar, C. A., \& Green, M. F. (2009). Errorless learning for training individuals with schizophrenia at a community mental health setting providing work experience. Schizophrenia Bulletin, 35(4), 807-815.

Koegel, R. L., \& Rincover, A. (1976). Some detrimental effects of using extra stimuli to guide learning in normal and autistic children. Journal of Abnormal Child Psychology, 4, 59-71.

La Taille, Y. C. (1997). O erro na perspectiva piagetiana. In J. A. Aquino (Ed.), Erro e fracasso na escola: Alternativas teóricas e práticas (pp. 25 61). São Paulo, SP: Summus.

Lancioni, G. E., \& Smeets, P. M. (1986). Procedures and parameters of errorless discrimination training with developmentally impaired individuals. International Review of Research in Mental retardation, 14, 135-164.

Lawrence, D. H. (1952). The transfer of a discrimination along a continuum. Journal of Comparative and Physiological Psychology, 45, 511-516.

Lovaas, O. I., Koegel, R. L., \& Schreibman, L. (1979). Stimulus overselectivity in autism: A review of research. Psychological Bulletin, 86(6), 1236-1254.

Lovaas, O. I., \& Schreibman, L. (1971). Stimulus overselectivity of autistic children in a two stimulus situation. Behavioral Research \& Therapy, 9, 305-310.

Macedo, L. (1994). Ensaios construtivistas (2. ed.). São Paulo, SP: Casa do Psicólogo.

Macedo, L. (1995). Para uma psicopedagogia construtivista. In E. S. Alencar (Ed.), Novas contribuições da psicologia aos processos de ensino e aprendizagem (pp. 119-140). São Paulo, SP: Cortez.

Matos, M. A. (1981). O controle de estímulos sobre o comportamento. Psicologia, 2, 1-15.

Matos, M. A. (1995). Análise de contingências no aprender e no ensinar. In E. S. Alencar (Ed.), Novas contribuições da psicologia aos processos de ensino e aprendizagem (pp. 143-165). São Paulo, SP: Cortez.

McCartney, L. L. A., \& LeBlanc, J. M. (1997). Errorless learning in educational environments: Using criterion-related cues to reduce errors. In D. M. Baer \& E. M. Pinkston (Eds.), Environment and behavior (pp. 80-96). Cumnor Hill, UK: Westview Press.
McIlvane, W. J., \& Dube, W. V. (1992). Stimulus control shaping and stimulus control topographies. The Behavior Analyst, 15, 89-94.

Melo, R. M., de Jesus, P. S., \& Hanna, E. S. (2005). Discriminação simples e comportamento conceitual de posição: Influência de diferentes tipos de treino. Revista Brasileira de Análise do Comportamento, 1, 231-252.

Mueller, M. M., Palkovic, C. M., \& Maynard, C. S. (2007). Errorless learning: Review and practical application for teaching children with pervasive developmental disorders. Psychology in the Schools, 44, 691-700.

Ostryn, C., \& Wolfe, P. S. (2011). Teaching preschool children with autism spectrum disorders to expressively discriminate between "what's that?" and "where is it?" Focus on Autism and Other Developmental Disabilities, 26, 195-205.

Piaget, J. (1976). Le possible, l'impossible et le nécessaire. Archives de Psychologie, 44, 281-299.

Pierce, W. B., \& Epling, W. F. (1995). Behavior analysis and learning. Englewood Cliffs, NJ: Prentice Hall.

Pinto, N. B. (2000). O erro como estratégia didática: Estudo do erro no ensino da matemática elementar. São Paulo, SP: Papirus.

Postalli, L. M. M., Almeida, D. M. B., Canovas, D. S., \& de Souza, D. G. (2008). Ensino de reconhecimento de palavras no contexto da leitura de histórias infantis. Revista Brasileira de Análise do Comportamento, 4, 27-51.

Queiroz, S. S., Dias, L. P., Chagas, J. D., \& Nepomoceno, P. S. (2011). Erros e equilibração em Psicologia Genética. Revista Semestral da Associação Brasileira de Psicologia Escolar e Educacional, 15, 263-271.

Raben, M. W. (1949). The white rat's discrimination of differences in intensity of illumination measured by a running response. Journal of Comparative Physiology and Psychology, 42, 254272.

Raulston, T., Carnett, A., Lang, R., Tostanoski, A., Lee, A., Machalicek, W., ...Lancioni, G. E. (2013). Teaching individuals with autism spectrum disorder to ask questions: A systematic review. Research in Autism Spectrum Disorders, 7, 866-876

Rincover, A. (1978). Variables affecting stimulus fading and discriminative responding in pshy- 
chotic children. Journal of Abnormal Psychology, 87, 541-553.

Schilmoeller, G. L., Schilmoeller, K. J., Etzel, B. C., \& LeBlanc, J. M. (1979). Conditional discrimination after errorless and trial-and-error training. Journal of the Experimental Analysis of Behavior, 31, 405-420.

Schlosberg, H., \& Solomon, R. (1943). Latency of response in a choice discrimination. Journal of Experimental Psychology, 33, 22-39.

Schreibman, L. (1975). Effects of within-stimulus and extra-stimulus prompting on discrimination learning in autistic children. Journal of Applied Behavior Analysis, 8, 91-112.

Schreibman, L., \& Charlop, M. H. (1981). S+ versus $\mathrm{S}-$ Fading in prompting procedures with autistic children. Journal of Experimental Child Psychology, 31, 508-520.

Schwartz, S. H., Firestone, I. J., \& Terry, S. (1971). Fading techniques and concept learning in children. Psychonomic Science, 25, 83-84.

Sério, T. M. A. P., Andery, M. A., Gioia, P. S., \& Micheletto, N. (2002). Controle de estímulos e comportamento operante: Uma introdução. São Paulo, SP: EDUC.

Sidman, M. (1985). Aprendizagem-sem-erros e sua importância para o ensino do deficiente mental. Psicologia, 11, 1-15.

Sidman, M. (2010). Errorless learning and programmed instruction: The myth of learning curve. European Journal of Behavior Analysis, 11, 167-180.

Sidman, M., \& Stoddard, L. T. (1966). Programming perception and learning for retarded children. In N. R. Ellis (Ed.), International review of research in mental retardation (Vol. 2, pp. 151-208). New York: Academic Press.

Sidman, M., \& Stoddard, L. T. (1967). The effectiveness of fading in programming a simultaneous form discrimination for retarded children. Journal of the Experimental Analysis of Behavior, 10, 3-15.

Skinner, B. F. (1938). The behavior of organisms: An experimental analysis. New York: Appleton-Century-Crofts.

Skinner, B. F. (1972). Tecnologia do ensino (R. Azzi, Trad.). São Paulo, SP: Herder. (Original publicado em 1968)
Smeets, P. M., \& Striefel, S. (1990). Discrimination training of mirror-image stimuli with a delayedprompt technique: Some critical dimensions of extra-stimulus prompts. Journal of Experimental Child Psychology, 49, 275-299.

Spence, K. W. (1937). The differential response in animals to stimuli varying within a single dimension. Psychological Review, 44, 430-444.

Stella, M. E., \& Etzel, B. C. (1986). Stimulus control of eye orientations: Shaping $\mathrm{S}+$ only versus shaping S- only. Analysis and Intervention in Developmental Disabilities, 6, 137-153.

Stoddard, L. T., de Rose, J. C., \& McIlvane, W. J. (1986). Observações curiosas acerca do desempenho deficiente após a ocorrência de erros. Psicologia, 12, 1-18.

Stoddard, L. T., McIlvane, W. J., \& de Rose, J. C. (1987). Transferência de controle de estímulo com estudantes deficientes mentais: Modelagem de estímulo, superposição e a aprendizagem em uma tentativa. Psicologia, 13, 13-27.

Strand, S. C. (1989). S+ versus S- fading in teaching visual discriminations to severely mentally handicapped children. Journal of Mental Deficiency Research, 33, 283-299.

Strand, S. C., \& Morris, R. C. (1988). Criterion-related versus non-criterion-related prompt training with severely mentally handicapped children. Journal of Mental Deficiency Research, 32, 137151.

Striefel, S., Wetherby, B., \& Karlan, G. R. (1979). Establishing generalized verb-noun instruction-following skills in retarded children. Journal of Experimental Child Psychology, 22, 247260 .

Stromer, R. (1979). Developing discriminative behaviors prerequisite for comunication: Contributions of operant research in stimulus control. In J. E. Button, T. C. Lovitti, \& T. D. Rowland (Eds.), Communications research in learning disabilities and mental retardation (pp. 191-233). Baltimore, MD: University Park Press.

Tabler, J. I., Glaser, R., \& Schaefer, H. H. (1965). Learning and programmed instruction. Boston, MA: Addison-Wesley.

Terrace, H. S. (1963). Discrimination learning with and without "errors". Journal of the Experimental Analysis of Behavior, 1, 1-27. 
Thorndike, E. L. (1911). Animal intelligence. Retrieved from http://psychclassics.yorku.ca/Thorndi$\mathrm{ke} /$ Animal/chap2.htm

Touchette, P. E. (1971). Transfer of stimulus control measuring the moment of transfer. Journal of the Experimental Analysis of Behavior, 15, 347354.

Touchette, P. E., \& Howard, J. S. (1984). Errorless learning: Reinforcement contingencies and stimulus control transfer in delayed prompting. Journal of Applied Behavior Analysis, 17, 175188.

Van Laarhoven, T., Johnson, J. W., Repp, A. C., Karsh, K. G., \& Lenz, M. (2003). Discrimination training: A comparison of two procedures for presenting multiple examples within a fading and non-fading paradigm. Research in Developmental Disabilities, 24, 1-18.
Warmington, M., Hitch, G. J., \& Gathercole, S. E. (2013). Improving word learning in children using an errorless technique. Journal of Experimental Child Psychology, 114, 456-465.

Zawlocki, R. J., \& Walls, R. T. (1983). Fading on the $\mathrm{S}+$, the S-, both, or neither. American Journal of Mental Deficiency, 87, 462-464.

Recebido: 31/05/2013

$1^{a}$ revisão: 19/09/2013

$2^{a}$ revisão: $22 / 10 / 2013$

Aceite final: 22/10/2013 